\title{
Exigência de metionina + cistina para frangas de reposição na fase recria de 13 a 18 semanas de idade
}

\author{
[Requirement of methionine plus cystine for egg pullets in the growing \\ phase from 13 to 18 weeks old] \\ P. D'Agostini, P.C. Gomes, H.H.C. Mello, A.A. Calderano, L.M. Sá, \\ H.S. Rostagno, L.F.T. Albino
}

Universidade Federal de Viçosa - Viçosa, MG

\begin{abstract}
RESUMO
Determinou-se a exigência de metionina + cistina para frangas de 13 a 18 semanas de idade e verificou-se seu efeito sobre a fase de postura. $\mathrm{O}$ experimento foi dividido em duas fases experimentais, recria e postura. Na fase de recria, 560 aves - 50\% Lohmann LSL e 50\% Lohmann Brown -, com 13 a 18 semanas de idade, foram distribuídas em delineamento inteiramente ao acaso, em esquema fatorial $5 \times 2$ (porcentagem de met+cis e linhagens), com quatro repetições e 14 aves por repetição. As porcentagens de met+cis totais estudadas foram 0,$399 ; 0,469 ; 0,539 ; 0,609$ e $0,679 \%$. Ao atingirem 22 semanas de idade, 240 aves foram utilizadas para se avaliar o efeito residual das dietas oferecidas na fase de recria sobre o desempenho das aves, a produção e a qualidade de ovos. Ao se considerar a fase de produção, não se verificou efeito da inclusão de níveis de met+cis na ração, para ambas as linhagens. As exigências de metionina + cistina totais foram de 0,679\% (0,611\% de met+cis digestível) para aves leves e de $0,646 \%$ $(0,581 \%$ de met+cis digestível) para aves semipesadas.
\end{abstract}

Palavras-chave: aminoácidos, ovo, maturidade sexual, poedeiras, postura

\begin{abstract}
The methionine + cystine requirement was determined for pullets from 13 to 18 weeks of age and its effect was verified on the laying phase. The experiment was divided into two experimental phases. In the growing phase 560 birds (50\% and 50\% Lohmann LSL Lohmann Brown) at 13 to 18 weeks of age were distributed in a completely randomized factorial scheme $5 \times 2$ (methionine + cystine levels and lineages), with four replications and 14 birds per repetition. The levels of total methionine + cystine studied were $0.399,0.469,0.539,0.609$ and $0.679 \%$. When they reached 22 weeks of age, 240 birds were used to assess the residual effects of the diets offered in the growing phase on performance of poultry, egg production and quality. Considering the production phase, there was no effect of the inclusion of methionine + cystine levels in the diet for both strains. The total methionine and cysteine was $0.679 \%$ $(0.611 \%$ of digestible methionine + cystine $)$ for light birds and $0.646 \%(0.581 \%$ methionine + cystine digestible) for brown-egg pullets.
\end{abstract}

Keywords: amino acids, egg, hens, production, sexual maturity

\section{INTRODUÇÃO}

A maturidade sexual e o desempenho da poedeira estão diretamente relacionados com o desenvolvimento adequado da ave nas fases que antecedem a fase de produção. Isto ocorre porque fatores que interferem na idade da ave

Recebido em 22 de agosto de 2011

Aceito em 20 de julho de 2012

E-mail: priscila.agostini@technofeed.com.br e na maturidade sexual estão diretamente relacionados com o peso corporal e a uniformidade do lote na fase de recria.

O conhecimento da curva de crescimento das aves de reposição tem mostrado que $70 \%$ do peso adulto de uma poedeira deve ser atingido até 12 semanas de vida (Faria, 1998), 82\% até 15 
semanas, e $92 \%$ até 22 semanas, enquanto os $8 \%$ restantes devem ser depositados posteriormente (Kwakkel, 1992). Aves com peso abaixo do ideal da linhagem retardam o início da postura, reduzindo com isto a produção de ovos; em contrapartida, aves com excesso de peso entram em postura precocemente e produzem ovos pequenos, o que também não é viável. Segundo Leeson e Summers (1997), o peso corporal influencia diretamente a maturidade sexual; além disso, os autores preconizam a importância de maximizar o ganho de peso.

A metionina é considerada o primeiro aminoácido limitante para aves, sendo, portanto, de fundamental importância para os desenvolvimentos corporal e reprodutivo das aves. Com o intuito de garantir o adequado desenvolvimento das aves poedeiras, faz-se necessário atualizar constantemente as exigências em metionina + cistina, tanto para aves leves como para semipesadas. Atualmente, com a utilização de metionina + cistina na forma sintética, os nutricionistas podem formular dietas com níveis mais baixos de proteína bruta. Com isto, as exigências nutricionais das aves são mais bem atendidas e há menor excreção de nitrogênio para o meio ambiente.

As estimativas da exigência nutricional de metionina + cistina para frangas de reposição, na fase de recria e pré-postura, são escassas. Além disso, com a constante seleção genética, o padrão de crescimento das aves é alterado, e, por consequência, as exigências nutricionais das aves podem ser modificadas.

Desta forma, objetivou-se determinar as exigências em aminoácidos sulfurosos metionina + cistina de poedeiras leves e semipesadas de 13 a 18 semanas de idade e avaliar o efeito desses níveis sobre o rendimento produtivo das aves na fase de produção.

\section{MATERIAL E MÉTODOS}

Para a determinação da exigência de metionina + cistina para frangas de reposição de 13 a 18 semanas de idade das linhagens comerciais Lohmann LSL e Lohmann Brown, o experimento foi dividido em duas fases experimentais, sendo a primeira a de recria, de
13 a 18 semanas de idade, em que se avaliaram as características de desempenho - consumo de ração, conversão alimentar e ganho de peso -, e a segunda a de produção, de 22 a 33 semanas de idade, em que se avaliaram as características de desempenho - consumo de ração, conversão alimentar e porcentagem de postura - e a qualidade do ovo.

Até atingirem as 13 semanas de idade, as aves foram criadas em galpões de recria e receberam ração e água à vontade. A ração foi feita de acordo com a exigência de cada fase - de zero a seis e de sete a 12 semanas de idade - de acordo com as recomendações de Rostagno et al. (2000). Para prevenção do vício de canibalismos, todas as aves foram debicadas aos 10 dias de idade. Além disso, foi adotado o manejo de vacinação para controle das principais enfermidades presentes nas aves de postura. Ao atingirem a idade de 13 semanas, as aves foram transferidas para as instalações experimentais, onde foram pesadas e alojadas em gaiolas suspensas (duas aves por gaiola), compostas com bebedouro tipo calha e comedouro. $O$ peso médio, por ave, no início do experimento foi de $831 \mathrm{~g}$ para frangas leves e de $1.018 \mathrm{~g}$ para semipesadas.

Quinhentas e sessenta aves de reposição fêmeas, 280 aves leves da marca comercial Lohmann LSL e 280 aves semipesadas da marca comercial Lohmann Brown foram distribuídas em delineamento experimental inteiramente ao acaso, em esquema fatorial $5 \times 2$, sendo cinco porcentagens de inclusão de met+cis e duas linhagens, com quatro repetições e 14 aves por unidade experimental.

As dietas experimentais foram obtidas a partir de uma dieta basal (Tab. 1) suplementada com cinco porcentagens de DL-Metionina (99\%), de forma a proporcionar 0,$399 ; 0,469 ; 0,539 ; 0,609 \mathrm{e}$ $0,679 \%$ de met+cis total. A suplementação com DL-Metionina foi feita em substituição ao amido, assegurando com isso que as rações permanecessem isoproteicas e isoenergéticas. Os demais nutrientes contidos nas rações foram utilizados atendendo às recomendações de Rostagno et al. (2000). A água e a ração foram fornecidas à vontade. 
Exigência de metionina...

Tabela 1. Composição percentual da dieta basal e valor nutricional para frangas de reposição leves e semipesadas de 13 a 18 semanas de idade

\begin{tabular}{|c|c|}
\hline Ingrediente & $\%$ \\
\hline Raspa de mandioca & 44,20 \\
\hline Sorgo & 22,28 \\
\hline Farelo de soja & 20,58 \\
\hline Farelo de trigo & 6,70 \\
\hline Óleo vegetal & 2,40 \\
\hline Fosfato bicálcico & 1,23 \\
\hline Calcário & 0,95 \\
\hline Sal & 0,27 \\
\hline Suplemento vitamínico ${ }^{1}$ & 0,10 \\
\hline Suplemento mineral ${ }^{2}$ & 0,05 \\
\hline Antioxidante $^{3}$ & 0,01 \\
\hline Virginamicina $50 \%$ & 0,002 \\
\hline Cloreto de colina $60 \%$ & 0,042 \\
\hline Salinomicina $12 \%^{4}$ & 0,035 \\
\hline Amido & 1,151 \\
\hline Total & 100 \\
\hline \multicolumn{2}{|l|}{ Composição } \\
\hline Energia metabolizável, kcal/ kg & 2.900 \\
\hline Proteína bruta, $\%$ & 15,1 \\
\hline Metionina + cistina total,$\%$ & 0,399 \\
\hline Metionina + cistina digestível, \% & 0,344 \\
\hline Lisina, $\%$ & 0,703 \\
\hline Cálcio, \% & 0,811 \\
\hline Fósforo disponível, \% & 0,319 \\
\hline
\end{tabular}

A temperatura no galpão foi monitorada três vezes ao dia, por meio de três termômetros distribuídos ao longo do galpão, posicionados à altura das aves. As temperaturas médias mínima e máxima foram 21 e $29^{\circ} \mathrm{C}$, respectivamente.

Ao final do período experimental, as aves permaneceram nas gaiolas, receberam ração à vontade, formulada de acordo com as recomendações de Rostagno et al. (2000). Nesta fase, iniciou-se o programa de luz, quando as aves receberam, inicialmente, 15 horas de luz; ao atingirem 20 semanas de idade receberam 16 horas de luz, e, ao completarem 22 semanas de idade, 17 horas de luz. O aumento do período de luz teve como objetivo assegurar que todas as aves entrassem em maturidade sexual e em produção de ovos na mesma época.
Ao atingirem 22 semanas de idade, iniciou-se a segunda fase experimental, sendo utilizadas 120 frangas da marca comercial Lohmann LSL e 120 da marca comercial Lohmann Brown, provenientes da primeira fase experimental. O delineamento utilizado foi inteiramente ao acaso, em esquema fatorial $5 \times 2$, sendo cinco porcentagens de inclusão de aminoácidos sulfurados e dois tipos de aves, leves e semipesadas, com quatro repetições e oito aves por unidade experimental. A ração fornecida nesta fase foi a mesma para todos os tratamentos, pois o objetivo era avaliar o efeito das dietas fornecidas no período de 13 a 18 semanas de idade. A ração contendo $2.800 \mathrm{kcal} / \mathrm{kg}$ de EM, $16 \%$ de $\mathrm{PB}, 4,02 \%$ de $\mathrm{Ca}$ e $0,377 \%$ de $\mathrm{Pd}$ atendeu às exigências nutricionais das aves, segundo as recomendações de Rostagno et al. (2000). 
O programa de luz adotado foi o preconizado pelo manual da linhagem, em que se manteve o fornecimento constante de 17 horas de luz durante todo o período experimental.

As temperaturas médias mínima e máxima no galpão foram, respectivamente, 14,4 e $28,8^{\circ} \mathrm{C}$.

$\mathrm{Na}$ fase de produção, foram avaliados os efeitos da inclusão de met+cis fornecidos no período de 13 a 18 semanas de idade sobre as características produtivas. As variáveis avaliadas foram: média, em dias, em que as aves atingiram $50 \%$ de produção em cada parcela experimental, produção de ovos, consumo alimentar, conversão alimentar, peso médio dos ovos, peso médio dos componentes dos ovos e unidade Haugh (UH).

Análises de regressão polinomial e Linear Response Plateau (LRP) foram usadas conforme o ajustamento dos dados obtidos para cada variável, utilizando-se o programa SAEG (Sistema..., 1999).

\section{RESULTADOS E DISCUSSÃO}

O ganho de peso, o consumo de ração e a conversão alimentar das aves na fase de recria foram influenciados pela porcentagem de inclusão de met+cis na ração (Tab. 2). Houve interação significativa entre porcentagem de met+cis na ração e linhagem das aves. Desta forma, aves leves e semipesadas exigiram quantidades diferentes de met+cis para melhorar as características produtivas.

O ganho de peso das aves leves e semipesadas foi melhor com a inclusão de $0,558 \%$ e $0,624 \%$ de met+cis na ração, respectivamente (Tab. 3). Portanto, as aves semipesadas têm maior exigência de met+cis para suportar o ganho de peso em relação às aves leves.

Tabela 2. Características de desempenho de frangas de reposição leves (L) e semipesadas (SP), no período de 13 a 18 semanas de idade, em razão da porcentagem de metionina + cistina nas rações

\begin{tabular}{|c|c|c|c|c|c|c|}
\hline \multirow{3}{*}{$\begin{array}{c}\text { Met+cis } \\
(\%)\end{array}$} & \multicolumn{6}{|c|}{ Características } \\
\hline & \multicolumn{2}{|c|}{ Ganho de peso (g) } & \multicolumn{2}{|c|}{ Consumo de ração $(\mathrm{g})$} & \multicolumn{2}{|c|}{ Conversão alimentar } \\
\hline & $\mathrm{L}$ & SP & $\mathrm{L}$ & SP & $\mathrm{L}$ & SP \\
\hline 0,399 & 326 & 378 & 1,997 & 2,297 & 6,12 & 6,07 \\
\hline 0,469 & 339 & 453 & 1,988 & 2,459 & 5,87 & 5,43 \\
\hline 0,539 & 390 & 459 & 2,032 & 2,366 & 5,22 & 5,15 \\
\hline 0,609 & 403 & 514 & 1,984 & 2,405 & 4,93 & 4,68 \\
\hline 0,679 & 386 & 522 & 1,915 & 2,312 & 4,96 & 4,43 \\
\hline Média & $369 b$ & $465 a$ & $1,983 b$ & $2,368 \mathrm{a}$ & $5,42 \mathrm{a}$ & $5,15 b$ \\
\hline & & & Efeito & & & \\
\hline Linear & ns & $*$ & ns & $\mathrm{ns}$ & $*$ & $*$ \\
\hline Quadrático & $*$ & ns & $* *$ & $*$ & ns & ns \\
\hline LRP & $* *$ & $* *$ & ns & ns & ns & $*$ \\
\hline Interação & \multicolumn{2}{|c|}{$*$} & \multicolumn{2}{|c|}{$* *$} & \multicolumn{2}{|c|}{$* *$} \\
\hline $\mathrm{CV}(\%)$ & \multicolumn{2}{|c|}{2,13} & \multicolumn{2}{|c|}{2,64} & \multicolumn{2}{|c|}{2,17} \\
\hline
\end{tabular}

Valores seguidos por letras diferentes na mesma linha diferem entre si pelo teste $\mathrm{F}(\mathrm{P}<0,05)$.

$*(\mathrm{P}<0,01) ; * *(\mathrm{P}<0,05) ;$ ns $(\mathrm{P}>0,05)$.

LRP: Linear Response Platô. 
Exigência de metionina...

Tabela 3. Equações de regressão dos modelos quadrático e linear, equações linear do modelo linear response platô (LRP) e exigências nutricionais estimadas

\begin{tabular}{|c|c|c|c|c|}
\hline Parâmetro & Equação & Exigência & $\mathrm{R}^{2}$ & SQD \\
\hline \multicolumn{5}{|c|}{ Leves } \\
\hline \multicolumn{5}{|l|}{ Quadrático } \\
\hline Ganho de Peso & $Y=-167,3+1777,6 x-1406 x 2$ & 0,634 & 0,88 & 0,0006 \\
\hline Consumo de ração & $Y=1,2396+3,1102 x-3,1055 x 2$ & 0,501 & 0,83 & 0,0012 \\
\hline \multicolumn{5}{|l|}{ Linear } \\
\hline Conversão alimentar & $Y=7,93065-4,65784 x$ & $>0,679$ & 0,89 & 0,1258 \\
\hline \multicolumn{5}{|l|}{ Linear Response Platô } \\
\hline Ganho de peso & $y=162,087+401,28 x$ & 0,558 & 0,93 & 0,0003 \\
\hline \multicolumn{5}{|c|}{ Semipesadas } \\
\hline \multicolumn{5}{|l|}{ Quadrático } \\
\hline Consumo de ração & $Y=0,8378+5,9111 x-5,5141 \times 2$ & 0,536 & 0,58 & 0,0076 \\
\hline \multicolumn{5}{|l|}{ Linear } \\
\hline Ganho de peso & $Y=196,033+499,876 x$ & $>0,679$ & 0,91 & 0,012 \\
\hline Conversão alimentar & $Y=8,26569-5,77879 x$ & $>0,679$ & 0,98 & 0,0423 \\
\hline \multicolumn{5}{|l|}{ Linear Response Platô } \\
\hline Ganho de peso & $Y=152,563+592,563 x$ & 0,624 & 0,92 & 0,0008 \\
\hline Conversão alimentar & $Y=8,54905-6,38295 x$ & 0,646 & 0,97 & 0,0244 \\
\hline
\end{tabular}

SQD: Soma dos Quadrados dos Desvios.

A porcentagem de met+cis na ração que favoreceu o maior consumo de ração para as aves leves e semipesadas foi, respectivamente, $0,501 \%$ e $0,536 \%$.

A conversão alimentar das aves foi afetada pela porcentagem de met+cis na ração. À medida que se aumentou a quantidade de met+cis na ração das aves leves, a conversão alimentar diminuiu. Por outro lado, a conversão alimentar das aves semipesadas foi menor com a inclusão de $0,646 \%$ de met+cis na ração, considerando-se o ajuste dos dados segundo o modelo estatístico LRP.

As aves semipesadas, em média, ganharam $26,0 \%$ mais peso, consumiram $19,4 \%$ mais ração e tiveram conversão alimentar $5,0 \%$ menor do que as aves leves. Estes resultados sugerem que o aumento no ganho de peso das aves semipesadas pode ser devido à melhor utilização dos nutrientes da ração.

Arq. Bras. Med. Vet. Zootec., v.64, n.6, p.1691-1698, 2012
Os valores estimados de exigência em met+cis totais em frangas de reposição leves, na fase de recria (13 a 18 semanas de idade), foram de 0,558 e $0,679 \%$, respectivamente, para ganho de peso e conversão alimentar. As aves semipesadas, na fase de 13 a 18 semanas de idade, tiveram as exigências em met+cis estimadas em 0,624 e $0,646 \%$, respectivamente, para ganho de peso e conversão alimentar.

Considerando-se a digestibilidade de $90 \%$ para metionina + cistina (Rostagno et al., 2000), as exigências em met+cis digestíveis para frangas leves de reposição, na fase de 13 a 18 semanas de idade, foram estimadas em, respectivamente, 0,502 e $0,611 \%$ para ganho de peso e conversão alimentar; e nas aves semipesadas, 0,562 \% para ganho de peso e $0,581 \%$ para conversão alimentar.

Aves leves que receberam rações com $0,399 \%$ de met+cis tiveram a postura retardada e somente atingiram 50\% de produção com 161 dias de 
idade. Aquelas que receberam ração com níveis superiores a $0,469 \%$ de met+cis na fase de recria atingiram 50\% de produção aos 150 dias de idade. Entretanto, não houve efeito da porcentagem de met+cis na ração sobre a idade em que as aves semipesadas atingiram $50 \%$ de produção. As aves semipesadas atingiram 50\% de produção aos 150 dias de idade.

A idade em que as aves, leves e semipesadas, atingiram $50 \%$ de produção está de acordo com os manuais das linhagens (Manual..., 2001a,b), que indicam $50 \%$ de produção das aves, em média, entre 21 e 22 semanas de idade. Logo, pode-se verificar que a deficiência em aminoácidos sulfurosos durante a fase de 13 a 18 semanas de idade não foi suficiente para afetar a produção das aves, exceto para o nível de $0,399 \%$ para aves leves. Provavelmente isto se deve ao fato de que, durante as fases que antecedem a recria, as aves receberam rações com níveis ideais de todos os nutrientes, o que favoreceu o ótimo desenvolvimento corporal e reprodutivo.

Não houve efeito da porcentagem de met+cis na ração de recria $(\mathrm{P}>0,05)$ sobre o desempenho e a produção das aves na fase de postura (Tab. 4).

Tabela 4. Efeitos residuais dos níveis de met+cis na ração de poedeiras leves (L) e semipesadas (SP), no período de 13 a 18 semanas de idade, sobre os parâmetros de consumo de ração (CR), conversão por dúzia de ovos (CDO), conversão por massa de ovos (CMO) e porcentagem de ovos produzidos (POP)

\begin{tabular}{|c|c|c|c|c|c|c|c|c|}
\hline \multirow{3}{*}{$\begin{array}{c}\text { Met+cis } \\
(\%)\end{array}$} & \multicolumn{8}{|c|}{ Parâmetros } \\
\hline & \multicolumn{2}{|c|}{ CR } & \multicolumn{2}{|c|}{$\mathrm{CDO}$} & \multicolumn{2}{|c|}{ CMO } & \multicolumn{2}{|c|}{ POP } \\
\hline & $\mathrm{L}$ & SP & $\mathrm{L}$ & SP & $\mathrm{L}$ & $\mathrm{SP}$ & $\mathrm{L}$ & SP \\
\hline 0,399 & 100,0 & 104,04 & 1,33 & 1,34 & 1,87 & 1,84 & 91,2 & 93,2 \\
\hline 0,469 & 100,0 & 102,13 & 1,30 & 1,34 & 1,79 & 1,84 & 92,6 & 91,5 \\
\hline 0,539 & 102,01 & 104,09 & 1,29 & 1,33 & 1,77 & 1,82 & 95,2 & 93,8 \\
\hline 0,609 & 99,13 & 105,18 & 1,34 & 1,36 & 1,78 & 1,87 & 92,8 & 92,9 \\
\hline 0,679 & 101,23 & 103,92 & 1,29 & 1,33 & 1,81 & 1,80 & 93,9 & 93,8 \\
\hline Média & $100,47 b$ & $103,87 \mathrm{a}$ & $1,31 \mathrm{a}$ & $1,34 a$ & $1,80 \mathrm{a}$ & $1,83 a$ & 93,2 & $93,0 \mathrm{a}$ \\
\hline \multicolumn{9}{|c|}{ Efeito } \\
\hline Linear & ns & ns & ns & ns & ns & ns & ns & ns \\
\hline Quadrático & ns & ns & $\mathrm{ns}$ & ns & ns & $\mathrm{ns}$ & $\mathrm{ns}$ & ns \\
\hline Interação & \multicolumn{2}{|c|}{ ns } & \multicolumn{2}{|c|}{ ns } & \multicolumn{2}{|c|}{$\mathrm{ns}$} & \multicolumn{2}{|c|}{ ns } \\
\hline $\mathrm{CV}(\%)$ & \multicolumn{2}{|c|}{1,83} & \multicolumn{2}{|c|}{4,08} & \multicolumn{2}{|c|}{4,18} & \multicolumn{2}{|c|}{2,41} \\
\hline
\end{tabular}

Valores seguidos por letras diferentes na mesma linha diferem entre si pelo teste $\mathrm{F}(\mathrm{P}<0,05)$.

Embora durante a recria a quantidade de met+cis tenha influenciado o desempenho das aves, na fase de postura não houve efeito residual, ou seja, desde que as aves recebam dieta balanceada na fase de produção, aquelas aves que receberam dieta deficiente em aminoácidos sulfurosos têm a capacidade produtiva restabelecida na postura. Estes resultados podem ser explicados pela hipótese do ganho compensatório observado por Kwakkel et al. (1991), em que aves alimentadas à vontade com níveis adequados de lisina, nas fases subsequentes à fase inicial, não tiveram seu desempenho afetado durante a fase de produção.

O consumo de ração variou com a linhagem estudada, sendo que as aves semipesadas consumiram mais ração do que as aves leves.
A inclusão de met+cis na ração de recria não afetou o peso dos ovos, o peso e a porcentagem da gema, do albúmen e da casca e a unidade Haugh (Tab. 5, 6,7).

Observou-se efeito linear dos níveis de met+cis somente sobre o índice de albúmen $(\mathrm{P}<0,05)$ dos ovos das aves leves (Tab. 7).

Pode-se inferir que independentemente dos níveis utilizados na fase de recria, as aves leves e as semipesadas não tiveram a qualidade do ovo afetada na fase de produção.

Aves leves alimentadas com níveis mais elevados de met+cis durante a fase inicial de criação apresentaram maior número de ovos com defeito. Estas aves apresentaram maior peso 
corporal, o que favoreceu aumento no peso dos ovos, precocidade de início de postura, resultando em maior incidência de ovos sem casca e quebrados.

Nas aves semipesadas, não se observou este efeito, devido à menor variação de peso corporal ocorrida entre os tratamentos, pois houve uma variação de, no máximo, $1,93 \%$ entre os tratamentos para as aves semipesadas e uma variação de até $4,76 \%$ entre os tratamentos das aves leves.

Em resumo, a inclusão de met+cis na fase de recria afeta as características produtivas das aves nesta idade, mas não mantém seus efeitos nas aves na fase de postura. Isso sugere que aves alimentadas com deficiência de met+cis na fase de recria podem recuperar seu desempenho produtivo caso recebam dieta adequada na fase de produção.

Tabela 5. Efeitos residuais dos níveis de met+cis na ração de poedeiras leves (L) e semipesadas (SP), no período de 13 a18 semanas de idade, sobre a qualidade dos ovos

\begin{tabular}{|c|c|c|c|c|c|c|}
\hline \multirow{3}{*}{$\begin{array}{l}\text { Met+cis } \\
(\%)\end{array}$} & \multicolumn{6}{|c|}{ Parâmetros } \\
\hline & \multicolumn{2}{|c|}{$\%$ gema } & \multicolumn{2}{|c|}{$\%$ albúmen } & \multicolumn{2}{|c|}{$\%$ casca } \\
\hline & $\mathrm{L}$ & SP & $\mathrm{L}$ & SP & $\mathrm{L}$ & SP \\
\hline 0,399 & 25,7 & 24,1 & 63,3 & 65,2 & 11,0 & 10,7 \\
\hline 0,469 & 25,2 & 23,6 & 63,8 & 65,5 & 11,0 & 10,9 \\
\hline 0,539 & 25,3 & 24,1 & 63,7 & 65,2 & 11,0 & 10,7 \\
\hline 0,609 & 25,0 & 24,3 & 64,0 & 64,7 & 11,0 & 10,9 \\
\hline 0,679 & 6,75 & 25,4 & 24,2 & 63,7 & 10,9 & 10,9 \\
\hline Média & $25,3 a$ & $24,1 b$ & $63,7 a$ & $65,1 b$ & $11,0 \mathrm{a}$ & $10,8 \mathrm{a}$ \\
\hline \multicolumn{7}{|c|}{ Efeito } \\
\hline Linear & ns & ns & ns & ns & ns & ns \\
\hline Quadrático & ns & ns & ns & ns & $\mathrm{ns}$ & ns \\
\hline Interação & \multicolumn{2}{|c|}{ ns } & \multicolumn{2}{|c|}{ ns } & \multicolumn{2}{|l|}{ ns } \\
\hline $\mathrm{CV}(\%)$ & \multicolumn{2}{|c|}{3,83} & \multicolumn{2}{|c|}{1,78} & \multicolumn{2}{|l|}{3,09} \\
\hline
\end{tabular}

Valores seguidos por letras diferentes na mesma linha diferem entre si pelo teste $\mathrm{F}(\mathrm{P}<0,05)$.

Tabela 6. Efeitos residuais dos níveis de met+cis na ração de poedeiras leves (L) e semipesadas (SP), no período de 13 a18 semanas de idade, sobre a qualidade dos ovos

\begin{tabular}{|c|c|c|c|c|c|c|c|c|}
\hline \multirow{3}{*}{$\begin{array}{l}\text { Met+cis } \\
(\%)\end{array}$} & \multicolumn{8}{|c|}{ Parâmetros } \\
\hline & \multicolumn{2}{|c|}{ Peso do ovo } & \multicolumn{2}{|c|}{$\begin{array}{l}\text { Peso da } \\
\text { gema }\end{array}$} & \multicolumn{2}{|c|}{$\begin{array}{l}\text { Peso do } \\
\text { albúmen }\end{array}$} & \multicolumn{2}{|c|}{$\begin{array}{c}\text { Peso da } \\
\text { casca }\end{array}$} \\
\hline & $\mathrm{L}$ & SP & $\mathrm{L}$ & SP & $\mathrm{L}$ & SP & $\mathrm{L}$ & SP \\
\hline 0,399 & 58,7 & 60,7 & 15,1 & 14,6 & 37,2 & 39,6 & 6,46 & 6,49 \\
\hline 0,469 & 60,1 & 60,5 & 15,2 & 14,3 & 38,4 & 39,7 & 6,62 & 6,60 \\
\hline 0,539 & 60,5 & 61,1 & 15,3 & 14,7 & 38,5 & 39,9 & 6,65 & 6,52 \\
\hline 0,609 & 60,0 & 60,4 & 15,0 & 14,7 & 38,4 & 39,1 & 6,59 & 6,60 \\
\hline 0,679 & 59,6 & 61,7 & 15,1 & 14,9 & 38,0 & 59,6 & 59,6 & 6,48 \\
\hline Média & $59,8 \mathrm{~b}$ & $60,9 a$ & $15,1 \mathrm{a}$ & $14,6 \mathrm{~b}$ & $38,1 \mathrm{~b}$ & $39,7 a$ & $6,56 a$ & $6,59 a$ \\
\hline \multicolumn{9}{|c|}{ Efeito } \\
\hline Linear & ns & ns & ns & ns & ns & ns & ns & ns \\
\hline Quadrático & ns & $\mathrm{ns}$ & $\mathrm{ns}$ & ns & ns & $\mathrm{ns}$ & ns & ns \\
\hline Interação & \multicolumn{2}{|c|}{ ns } & \multicolumn{2}{|c|}{ ns } & \multicolumn{2}{|c|}{ ns } & \multicolumn{2}{|c|}{ ns } \\
\hline $\mathrm{CV}(\%)$ & \multicolumn{2}{|c|}{2,48} & \multicolumn{2}{|c|}{3,26} & \multicolumn{2}{|c|}{3,80} & \multicolumn{2}{|c|}{3,04} \\
\hline
\end{tabular}

Valores seguidos por letras diferentes na mesma linha diferem entre si pelo teste $\mathrm{F}(\mathrm{P}<0,05)$.

Observou-se efeito linear dos níveis de met+cis somente sobre o índice de albúmen $(\mathrm{P}<0,05)$ dos ovos das aves leves (Tab. 7). 
Tabela 7. Efeitos residuais dos níveis de met+cis na ração de poedeiras leves (L) e semipesadas (SP), no período de 13 a 18 semanas de idade, sobre a unidade Haugh (UH), o índice de gema (IG) e o índice de albúmen (IA)

\begin{tabular}{|c|c|c|c|c|c|c|}
\hline \multirow{3}{*}{$\begin{array}{l}\text { Met+cis } \\
(\%)\end{array}$} & \multicolumn{6}{|c|}{ Parâmetros } \\
\hline & \multicolumn{2}{|c|}{$\mathrm{UH}$} & \multicolumn{2}{|c|}{ IG } & \multicolumn{2}{|c|}{ IA } \\
\hline & $\mathrm{L}$ & SP & $\mathrm{L}$ & SP & $\mathrm{L}$ & SP \\
\hline 0,399 & 96,6 & 91,7 & 4,74 & 4,76 & 1,39 & 1,21 \\
\hline 0,469 & 98,2 & 93,0 & 4,74 & 4,69 & 1,41 & 1,18 \\
\hline 0,539 & 94,3 & 93,8 & 4,64 & 4,76 & 1,33 & 1,20 \\
\hline 0,609 & 96,2 & 90,7 & 4,62 & 4,70 & 1,32 & 1,13 \\
\hline 0,679 & 95,8 & 93,5 & 4,65 & 4,71 & 1,29 & 1,21 \\
\hline Média & $96,2 \mathrm{a}$ & $92,5 b$ & $4,68 \mathrm{a}$ & $4,72 \mathrm{a}$ & $1,35 \mathrm{a}$ & $1,19 b$ \\
\hline \multicolumn{7}{|c|}{ Efeito } \\
\hline Linear & $\mathrm{ns}$ & $\mathrm{ns}$ & ns & ns & $* *$ & ns \\
\hline Quadrático & $\mathrm{ns}$ & $\mathrm{ns}$ & $\mathrm{ns}$ & ns & ns & ns \\
\hline LRP & $\mathrm{ns}$ & $\mathrm{ns}$ & $\mathrm{ns}$ & $\mathrm{ns}$ & $\mathrm{ns}$ & $\mathrm{ns}$ \\
\hline Interação & \multicolumn{2}{|c|}{ ns } & \multicolumn{2}{|c|}{ ns } & \multicolumn{2}{|c|}{$\mathrm{ns}$} \\
\hline $\mathrm{CV}(\%)$ & \multicolumn{2}{|c|}{2,96} & \multicolumn{2}{|c|}{2,08} & \multicolumn{2}{|c|}{7,44} \\
\hline
\end{tabular}

Valores seguidos por letras diferentes na mesma linha diferem entre si pelo teste $\mathrm{F}(\mathrm{P}<0,05)$.

\section{CONCLUSÃO}

Recomendam-se $0,679 \%$ de metionina+cistina total (correspondendo a $0,611 \%$ de met+cis digestível) para aves leves de reposição e $0,646 \%$ de metionina+cistina total (correspondendo a $0,581 \%$ de met+cis digestível) para aves semipesadas.

\section{REFERÊNCIAS}

KWAKKEL, R.P.; KONING, F.L.S.M.; VERSTEGEN, M.W.A. et al. Effect of method and phase of nutrient restriction during rearing on productive performance of light hybrid pullets and hens. Br. Poult. Sci., v.32, p.747-761, 1991.
LEESON, S.; SUMMERS. J.D. Commercial Poultry Nutrition. 2.ed. Canada: University Books. 1997. 350p

MANUAL de Criação e Manejo-Lohmann brown. Granja Planalto. 10.ed., 2001a. 22p.

MANUAL de Criação e Manejo-Lohmann, Lsl. Granja Planalto. 10.ed. 2001b, 22p.

ROSTAGNO, H.S.; ALBINO, L.F.T.; DONZELE, J.L. et al. Tabelas brasileiras para aves e suínos; composição de alimentos $e$ exigências nutricionais. 1.ed. Viçosa-MG: UFV, Departamento de Zootecnia, 2000. 141p.

SISTEMA de análises estatísticas e genéticas (SAEG). Versão 8.0. Viçosa, MG. UFV, 1999. 142p. 